\title{
Optimization of Pd Surface Plasmon Resonance sensors for hydrogen detection
}

\author{
C. Perrotton ${ }^{a}$, N. Javahiraly ${ }^{a}$, M. Slaman ${ }^{b, c}$, H. Schreuders ${ }^{b}$, B. Dam ${ }^{b}$ and P. Meyrueis ${ }^{a}$ \\ ${ }^{a}$ Laboratoire des systemes photoniques, Universite de Strasbourg, Pole API, Bvd Sebastien \\ Brant, 67400 Illkirch, France; \\ ${ }^{b}$ Delft University of Technology, Department of Chemical Engineering, Julianalaan 136, 2628 \\ BL Delft, The Netherlands; \\ ${ }^{c}$ Faculty of Sciences, Department of Physics and Astronomy, Condensed Matter Physics, Vrije \\ Universiteit, De Boelelaan 1081, 1081 HV Amsterdam, The Netherlands.
}

\begin{abstract}
A design to optimize a fiber optic Surface Plasmon Resonance (SPR) sensor using Palladium as a sensitive layer for hydrogen detection is presented. In this approach, the sensitive layer is deposited on the core of a multimode fiber, after removing the optical cladding. The light is injected in the fiber with a given wavelength and all fiber modes are equally excited. The intensity modulation at the fiber output is measured to estimate the presence of hydrogen absorbed by the Pd, and consequently the Hydrogen concentration in the environment. The sensor response depends on both the Transverse Magnetic (TM) polarization (magnetic field perpendicular to incidence plane) and the Transverse Electric (TE) polarization (electric field perpendicular to incidence plane). The response for the Transverse Electric polarization is opposite with respect to the Transverse Magnetic polarization. The objective here is to optimize the Pd-SPR hydrogen sensor design in order to increase its sensitivity. We introduce an analysis of the sensor response as a function of the Pd thickness. Finally, a new design based on a multilayer system is proposed to enhance the SP 'effect'.
\end{abstract}

Keywords: Fiber optic sensor, Hydrogen sensor, Surface Plasmon, Palladium.

\section{INTRODUCTION}

Optical fiber sensors are of great interest to hydrogen detection in an explosive environment (explosive atmosphere for concentrations between $4 \%$, the lower explosive limit and $74,5 \%$, the upper explosive limit, at room temperature and pressure). There is no risk of gas ignition since the fiber does not interact electrically with the environment: no wire, no spark and no heat exchange. Besides, its geometrical configuration (small dimensions, light weight, geometrical versatility), the remote sensing capability and the spatial distribution made it the best candidate for embedded system such as aerospace shuttles or automotive applications. Most optical hydrogen fiber sensors use $\mathrm{Pd}$ as a transducer. ${ }^{1-4}$ Upon hydrogenation, the Pd is transformed to a Pd hydride. ${ }^{5}$ During this reversible transformation, the $\mathrm{Pd}$ transducer is prone to physical change, such as its dielectric permittivity, lattice constant and conductivity. Surface plasmon resonance sensors are widely recognized for investigating surface interaction. They turn out to be serious candidates for hydrogen detection based on thin films. Chadwick et al. ${ }^{6}$ proposed to use the surface plasmon resonance in Pd to realize a hydrogen sensor operating in the 0.1-10\% $H_{2}$ concentration range. In continuation on the work of Bevenot et al. ${ }^{7}$, we presented a Pd-SPR multimode fiber hydrogen sensor where all modes are equally excited in the optical fiber for the TE and TM polarization in the $450-850 \mathrm{~nm}$ wavelength range. ${ }^{8}$ The sensor consisted of an 'average' $10 \mathrm{~nm}$ Pd film deposited on the fiber core. The sensor showed a continuous spectral response shift in the $0.5-4 \% \mathrm{H}_{2}$ concentration range.

We propose here a theoretical study in order to optimize the sensor sensitivity. After showing the influence of the Pd thickness on the sensor response, we will discuss the possible applications and the future directions to take in order to realize an efficient SPR hydrogen sensor.

Further author information: (Send correspondence to C. Perrotton)

E-mail: cedric.perrotton@etu.unistra.fr

SPIE Eco-Photonics 2011: Sustainable Design, Manufacturing, and Engineering Workforce Education for a Green Future, edited by Pierre Ambs, Dan Curticapean, Claus Emmelmann, Wolfgang Knapp, Zbigniew T. Kuznicki, Patrick P. Meyrueis, Proc. of SPIE Vol. 8065, 80651F · C 2011 SPIE · CCC code: 0277-786X/11/\$18 · doi: 10.1117/12.882518 


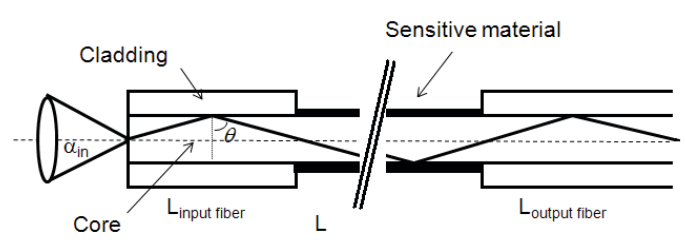

Figure 1. Hydrogen sensitive material deposited on the fiber core.

\section{SIMULATION MODEL}

In our simulation we consider multimode step index fibers. We simulate the multimode fiber SPR sensor in a planar approach and consider separately the TE and TM polarizations, ${ }^{9}$ (-so-called s and p, respectively-). The theory of local plane waves associated to a geometric optics approach is used. ${ }^{10}$ The transmitted intensity through the fiber is calculated by summing the power loss/gain at each reflection over the sensitive area. For that, the reflection coefficient is calculated as a function of angle by the transfer matrix method. ${ }^{11}$ The total transmitted power $I_{\text {out }}$ at a given wavelength $\lambda$ is defined as:

$$
I_{\text {out }, \lambda}=1 / 2\left(\int_{\theta_{c}}^{90}\left|r_{p}(\theta)\right|^{2 N} I_{0, \lambda}\left(\theta_{\text {in }}\right) d \theta+\int_{\theta_{c}}^{90}\left|r_{s}(\theta)\right|^{2 N} I_{0, \lambda}\left(\theta_{\text {in }}\right)\right) d \theta
$$

with:

$$
N=L /(D \times \tan \theta)
$$

where L, D, N are respectively the length of the sensitive area, the fiber diameter and the number of reflections along the sensor area, while $r_{s}, r_{p}$ are the reflection coefficient for polarization TE and TM, respectively. $\theta$ defines the angle between the normal of the reflection interface and the direction of the incident light. $\theta_{c}$ is the critical angle. $I_{0}$ defines the angular intensity distribution corresponding to the used light source. The distribution is assumed to be uniform simulating a long fiber length. Furthermore the TE and TM polarization are assumed to be equally distributed. Only meridian rays are considered. The model does not take into account scattering from possible roughness of the layer and mode coupling. The normalized intensity is defined as: $I_{\text {out }, \lambda} / \int_{\theta_{c}}^{90} I_{0}(\theta) d \theta$. We need the dielectric permittivity of the multilayer system to calculate the reflection coefficient. For this we used the $\mathrm{SiO}_{2}$ dielectric permittivity given by the Smeiller equation with the Schott industry coefficient as in. ${ }^{8}$ For Pd and Pd hydride the experimental dielectric permittivity as obtained by von Rottkay ${ }^{12}$ was used. The experimental data of the dielectric permittivity gives satisfying results with respect to the experimental sensor response reported. ${ }^{8}$ The $\mathrm{Pd}$ expansion during hydrogenation was neglected, which is legitimate for very thin films.

\section{RESULT}

The sensor response depends on the fiber parameter, the transducer layer and the power distribution. Fig. 2 shows the influence of the transducer length and the fiber NA. The sensor response is characterized by the transducer layer if the transducer numerical aperture (NA) is less than the fiber NA. We take therefore a large value for the fiber NA of 0.48 . Furthermore, the length of the transducer and the diameter of the fiber, which determine the number of reflection for each propagating ray, characterize the ratio between the tansmitted optical power and the optical power to be dissipated/coupled into the sensitive film for each propagating ray . We set the deposit length $\mathrm{L}$ at $2 \mathrm{~cm}$ and the fiber radius $\mathrm{r}$ at $100 \mu \mathrm{m}$ in order to focus only on the influence of the $\mathrm{Pd}$ thickness. It is worth noting that the light source, in this case, does not affect the response of the sensor, since we assume an uniform power distribution through the ray due to mode mixing, inherent to a non ideal fiber.

We calculate the relative transmission in the 3 - $15 \mathrm{~nm}$ thickness range for the TM-, TE- and both polarized light, as shown in FIG.3. The thickness range is chose such that fast hydrogen detection and a good reproducibility of the sensor occur. The response time is limited by the catalytic reaction. The degradation of $\mathrm{Pd}$ thin films over hydrogen cycling is less than one observed with thick films. The sensitivity of the sensor S defines the absolute change in intensity in presence of $4 \% \mathrm{H}_{2}$ in $\operatorname{Ar}\left(S=\mid\left(\right.\right.$ Iout $\left.\left.-I_{\text {out }, H_{2}}\right) \mid\right)$.

The losses for the TM polarized light decrease upon hydrogenation due to the narrowing of the Pd SPR peak. 

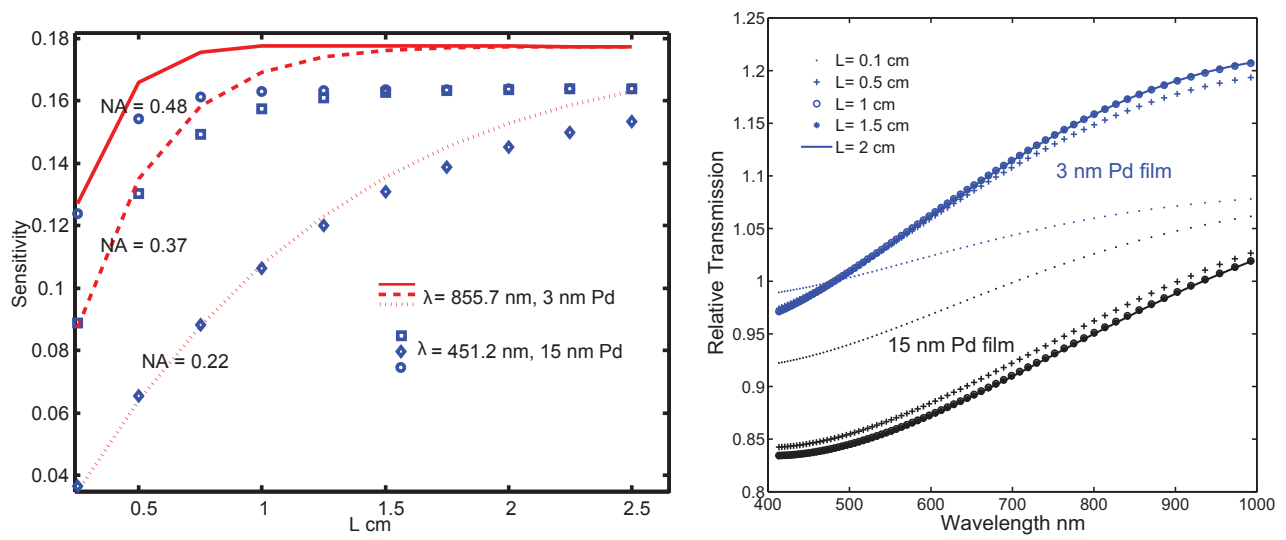

Figure 2. (a) Sensitivity as a function of the length of the Pd film deposited onto the fiber for different wavelengths and NA. (b) Relative transmission as a function of the wavelength for different lengths with a fiber NA of 0.48.
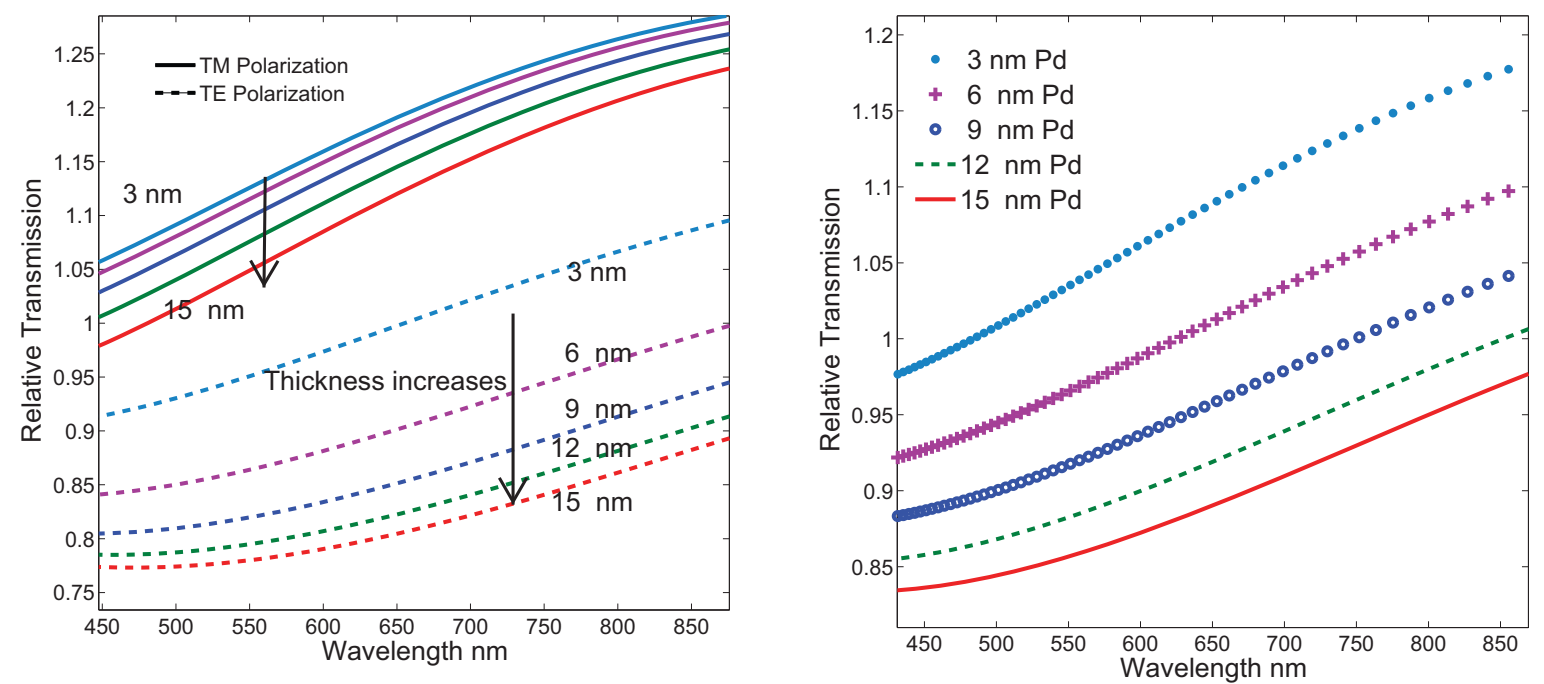

Figure 3. Relative Transmission as a function of the wavelength for different thickness of Pd (a) for p- (lines) and spolarization (symbols) and (b) for both polarizations.

Although the SPR peak is maximal for the $12 \mathrm{~nm} P d$ at a wavelength of $670 \mathrm{~nm}$, shown in fig.4, the maximum sensitivity is obtained for the $3 \mathrm{~nm}$ Pd film. The SPR occurs at a resonant angle outside the angle range for the allowed propagating ray (determined by the fiber NA). The sensitivity of the sensor for the TM polarization is related rather on the change in the width of the SPR peak than in the shift of the resonance.

The losses for the TE polarized light increase upon hydrogenation. These losses account for a more semiconductive like behaviour of the Pd film upon hydrogenation. The sensitivity increases with the thickness. Roughly speaking, the probe light sees more hydrogen absorbed in the Pd thick film than in the thinner film .

For the presented sensor, both TE and TM polarized light propagate through the fiber. The measure of the transmitted intensity of the both polarizations allows to detect the presence of hydrogen. Fig. $3 \mathrm{~b}$ shows the relative transmission of the sensor for $\mathrm{Pd}$ film in the 3-15 $\mathrm{nm}$ thickness range. The maximum sensitivity is obtained for a $3 \mathrm{~nm}$ Pd film and for a $15 \mathrm{~nm}$ Pd film below and above $665 \mathrm{~nm}$, respectively. As a result, the sensor response depends more on the TM polarization for thinner film. In contrast to thick films where the sensor response depends more on the TE polarization. To realize a sensor based on the SPR 'effect', the film has to be as thin as possible. Although the wavelength dependence of the sensor appears attractive for hydrogen sensing, the presented sensor does not take fully advantage of the SPR sensitivity. The SPR is in fact beyond the fiber NA and the competition in the response between the TE- and TM polarization decrease drastically the sensitivity of the SPR 'effect'. We have recently proposed a new design to couple the SP in the NA fiber 

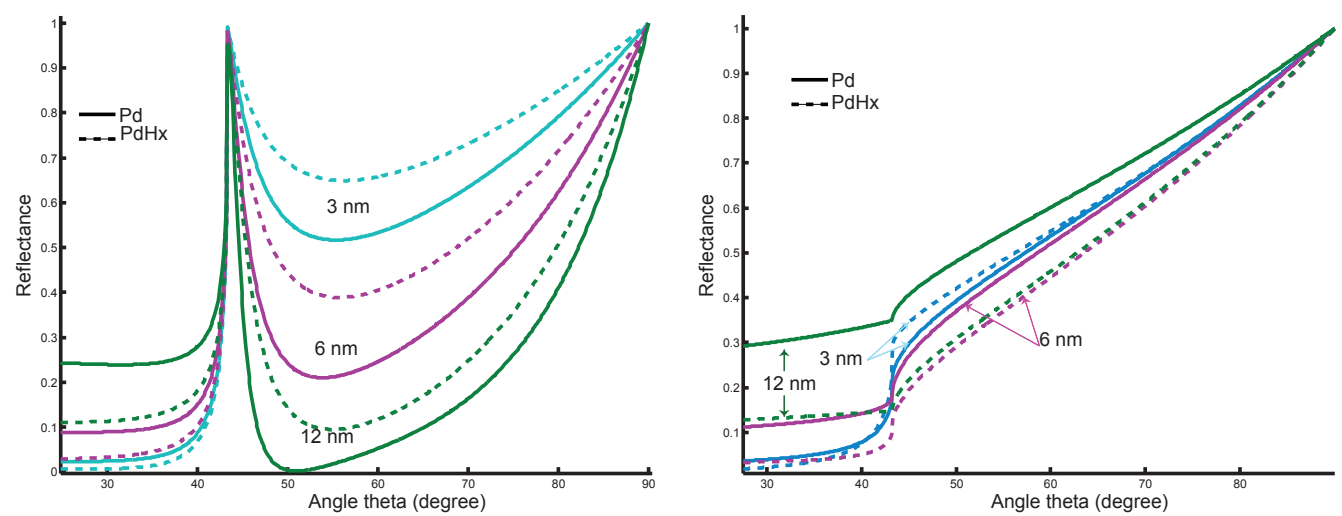

Figure 4. Reflectance of a $\mathrm{Pd}$ layer and a $P d H_{x}$ layer of $12 \mathrm{~nm}$ on a glass substrate as a function of angle theta $\left(0^{\circ}\right.$ corresponds to the normal incidence) for (a) p-polarization and (b) s-polarization. The used wavelength is $670 \mathrm{~nm}$.
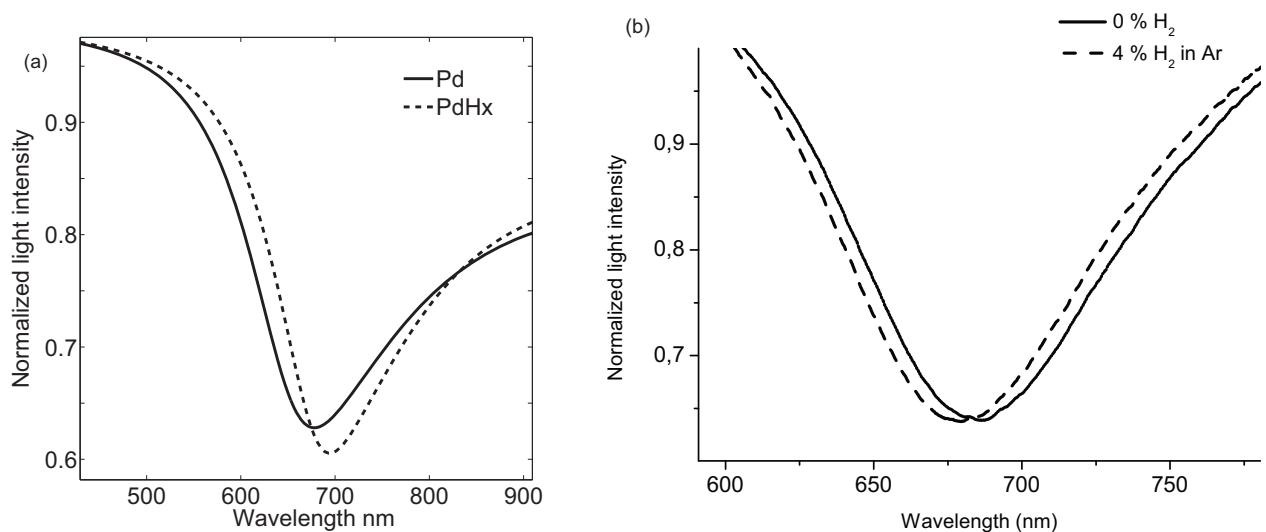

Figure 5. (a) The simlulated and (b) the experimental transmitted light intensity as a function of the wavelength. The transducer is a multilayer made of a $35 \mathrm{~nm}-\mathrm{Au} / 180 \mathrm{~nm}-\mathrm{SiO}_{2} / 3 \mathrm{~nm}-\mathrm{Pd}$ layer.

range. ${ }^{13}$ The $\mathrm{Pd}$ transducer film is replaced here by a multilayer stack made of a $\mathrm{Au}$, a $\mathrm{SiO}_{2}$ and a $\mathrm{Pd}$ layer. The hydrogen detection is based on the wavelength interrogation of the SPR peak. As shown in fig.5a, the spectral resonance shifts in the presence of $H_{2}$. The transmitted intensity shows an offset of 0.5. Since the multilayer design responds only to TM polarization, TE can be used as a reference signal. Our first experimental results confirm the SPR in the transmitted intensity. Fig.5b shows the spectral response for a multilayer made of 35 $\mathrm{nm}-\mathrm{Au} / 180 \mathrm{~nm}-\mathrm{SiO}_{2} / 3 \mathrm{~nm}-\mathrm{Pd}$. The SPR peak shifts in presence of $4 \% \mathrm{H}_{2}$ in Ar. We observe discrepancy between the simulated and the experimental results, in particular the shift of the SPR peak is opposite to the simulation. Further investigations have to been done in order to understand the spectral behavior of the sensor, as well as to determine the performance of this new sensor based on wavelength modulation (level of the minimum detection, sensitivity, reproducibility and response time).

\subsection{CONCLUSION}

The main advantage of the proposed Pd sensor is to follow the hydrogen concentration at several wavelengths. By comparing the intensity measured at different wavelengths, false alarms are avoided even if the signal is prone to intensity fluctuations. Besides, by varying the Pd thickness, we can optimize the sensitivity as a function of the operating wavelength. It turns out that the maximum sensitivity is obtained for a $3 \mathrm{~nm} P d$ film and for a $15 \mathrm{~nm}$ Pd film below and above $665 \mathrm{~nm}$ respectively. Moreover, siince each Pd film thickness presents its own spectral response, a low cost multi-point sensor is conceivable. However the realization of such a sensor with numerous multi-point sensing could turn out to be unreliable. Each sensitive section is modulated by the number of sensitive sections along the fiber. As each section responds continuously to the hydrogen concentration in a range of $0.5-4 \% \mathrm{H}_{2}$ and is modulated by all sensitive sections along the fiber, intensity fluctuation or polarization 
mixing could lead to false alarm. To get rid of the intensity fluctuation and the sensitivity of the sensor for TE polarized light, we propose to replace the $\mathrm{Pd}$ transducer by a multilayer stack made of $\mathrm{Au}, \mathrm{SiO}_{2}$ and $\mathrm{Pd}$. By coupling the SP in the fiber NA we succeed in realizing a SPR fiber sensor based on wavelength modulation. The next step is to replace the Pd by Pd nanoparticles, $W_{3}{ }^{14}$ and $\mathrm{Mg}$ alloys ${ }^{15}$ in order to increase the sensor sensitivity, decrease the cross-sensitivity and improve the sensor reproducibility.

\section{ACKNOWLEDGMENTS}

We acknowledge financial support from the Nederlandse Organisatie voor Wetenschappelijk Onderzoek NWO through the Sustainable Hydrogen Programme of Advanced Chemical Technologies for Sustainability program. The funding from the Region Alsace in France is gratefully acknowledged.

\section{REFERENCES}

[1] M.A. Butler, Optical fiber hydrogen sensor, Applied Physics Letters 45 (1984), no. 10, 1007-1009.

[2] J. Villatoro and D. Monzon-Hernandez, Fast detection of hydrogen with nano fiber tapers coated with ultra thin palladium layers, Optics Express 13 (2005), no. 13, 5087-5092.

[3] R.R.J. Maier, B.J.S. Jones, J.S. Barton, S. McCulloch, T. Allsop, J.D.C. Jones, and I. Bennion, Fibre optics in palladium-based hydrogen sensing, Journal of Optics A-Pure and Applied Optics 9 (2007), no. 6, S45-S59.

[4] X.T. Wei, T. Wei, H. Xiao, and Y.S. Lin, Nano-structured pd-long period fiber gratings integrated optical sensor for hydrogen detection, Sensors and Actuators B-Chemical 134 (2008), no. 2, 687-693.

[5] FA Lewis, Hydrogen in palladium and palladium alloys, International Journal of Hydrogen Energy 21 (1996), no. $6,461-464$.

[6] B. Chadwick, and M. Gal, Enhanced optical detection of hydrogen using the excitation of surface plasmons in palladium, Sensors and Actuators B: Chemical 123 (2007).

[7] X. Bevenot, A. Trouillet, C. Veillas, H. Gagnaire, and M. Clement, Surface plasmon resonance hydrogen sensor using an optical fibre, Measurement Science \& Technology 13 (2002), no. 1, 118-124.

[8] C. Perrotton, M. Slaman, N. Javahiraly, H. Schreuders, B. Dam, and Meyrueis, Wavelength response of a surface plasmon resonance palladium coated optic fiber, Optical Engineering 50 (2011).

[9] RC Jorgenson and SS Yee, A fiber-optic chemical sensor based on surface plasmon resonance, Sensors and Actuators B: Chemical 12 (1993), no. 3, 213-220.

[10] A.W. Snyder and J.D. Love, Optical waveguide theory, Springer, 1983.

[11] Abeles, La theorie generale des couches minces, Le journal de Physique et le Radium 11 (1950), 307-310.

[12] K. von Rottkay, M. Rubin, and P. A. Duine, Refractive index changes of pd-coated magnesium lanthanide switchable mirrors upon hydrogen insertion, Journal of applied physics 85 (1999), 408-413.

[13] C. Perrotton, N. Javahiraly, M. Slaman, B. Dam, and Meyrueis, A fiber optic Surface Plasmon Resonance sensor based on wavelength modulation for hydrogen sensing, submitted to Optics Express (2011).

[14] K. Ito and T. Ohgami, Hydrogen detection based on coloration of anodic tungsten-oxide film, Applied Physics Letters 60 (1992), no. 8, 938-940.

[15] M. Slaman, B. Dam, H. Schreuders, and R. Griessen, Optimization of mg-based fiber optic hydrogen detectors by alloying the catalyst, International Journal of Hydrogen Energy 33 (2008), no. 3, 1084-1089. 